\title{
Simultaneous Extraction, Fractionation and Enrichment of Microalgal Triacylglyerides by Exploiting the Tunability of Neat Supercritical Carbon Dioxide
}

\author{
Thomas A. Kwan ${ }^{a}$, Qingshi Tu ${ }^{a}$ and Julie B. Zimmerman ${ }^{a, b}$
}

\footnotetext{
${ }^{a}$ Department of Chemical and Environmental Engineering, Yale University, 9 Hillhouse Avenue, New Haven, CT, USA.

${ }^{\mathrm{b}}$ School of Forestry and Environmental Studies, Yale University, 195 Prospect St, New Haven, CT, USA. E-mail: Julie.Zimmerman@yale.edu, Tel: +1-203-432-9703

This six page supplement contains the following materials:

Figure S.1 Process flow diagram (PFD) of the downstream processing model

Table S.2 Operating conditions and solubilities of analytical standard and Chlorella sp. TAG extracts

Table S.3 Solubility separation factors
} 


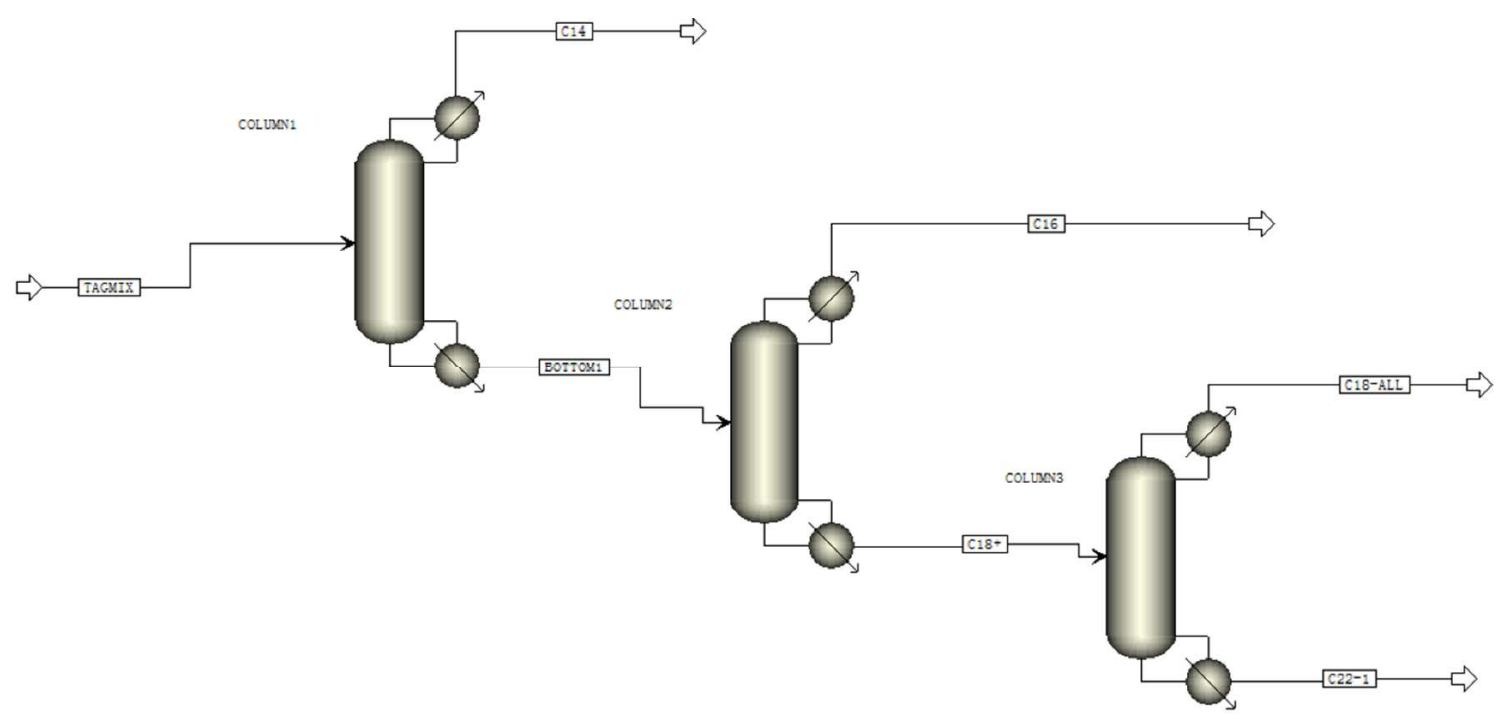

Figure S.1 Process flow diagram (PFD) for the downstream processing model

Table S.2 Measured solubility, $y^{*}$, and operational conditions for binary and multi-component analytical standard TAG mixtures and TAGs extracted from the microalgae Chlorella sp. (Note: extracts from Chlorella sp. were modeled to be a triacylglyceride with identical fatty acids and reported as the modeled chain length and saturation.)

\begin{tabular}{|c|c|c|c|c|c|c|}
\hline & $\begin{array}{l}T \\
{ }^{o} C\end{array}$ & $\begin{array}{c}P \\
M P a\end{array}$ & $\begin{array}{l}\text { Density } \\
m g / m L\end{array}$ & $\begin{array}{c}y^{*} \\
\text { solute/solvent }\end{array}$ & $\begin{array}{l}R^{2} \\
-\end{array}$ & $\begin{array}{c}\text { stdev } \\
-\end{array}$ \\
\hline & \multicolumn{6}{|c|}{ Binary Mixture of Analytical Standards } \\
\hline $\begin{array}{l}\text { C16:0 } \\
\text { C18:1 }\end{array}$ & 40 & 8.31 & $\begin{array}{c}384 \\
\text { (very low) }\end{array}$ & $\begin{array}{l}0.02 \\
0.06\end{array}$ & $\begin{array}{l}0.983 \\
0.999\end{array}$ & $\begin{array}{l}7.9 \mathrm{E}-03 \\
1.3 \mathrm{E}-03\end{array}$ \\
\hline $\begin{array}{l}\text { C16:0 } \\
\text { C18:1 }\end{array}$ & 40 & 13.4 & $\begin{array}{c}750 \\
\text { (high) }\end{array}$ & $\begin{array}{l}0.04 \\
0.03\end{array}$ & $\begin{array}{l}0.990 \\
0.987\end{array}$ & $\begin{array}{l}5.1 \mathrm{E}-03 \\
8.2 \mathrm{E}-03\end{array}$ \\
\hline
\end{tabular}

Multi-component Mixture of Analytical Standards

\begin{tabular}{|c|c|c|c|c|c|c|c|}
\hline Unsaturated & C14:0 & \multirow{6}{*}{40} & \multirow{6}{*}{9.3} & \multirow{6}{*}{$\begin{array}{c}550 \\
\text { (medium) }\end{array}$} & 0.06 & 0.997 & $1.9 \mathrm{E}-03$ \\
\hline \multirow{5}{*}{ MUFA } & C16:0 & & & & 0.04 & 0.923 & $9.3 \mathrm{E}-03$ \\
\hline & C18:0 & & & & 0.03 & 0.945 & $5.3 \mathrm{E}-03$ \\
\hline & C16:1 & & & & 0.11 & 0.987 & $1.6 \mathrm{E}-03$ \\
\hline & C18:1 & & & & 0.05 & 0.995 & $1.4 \mathrm{E}-03$ \\
\hline & C20:1 & & & & 0.11 & 0.996 & $1.3 \mathrm{E}-03$ \\
\hline \multirow[t]{2}{*}{ Unsaturated } & C14:0 & \multirow{2}{*}{40} & \multirow{2}{*}{13.35} & \multirow{2}{*}{$\begin{array}{c}750 \\
\text { (high) }\end{array}$} & 0.11 & 0.989 & 2.7E-03 \\
\hline & C16:0 & & & & 0.07 & 0.951 & 4.9E-03 \\
\hline
\end{tabular}




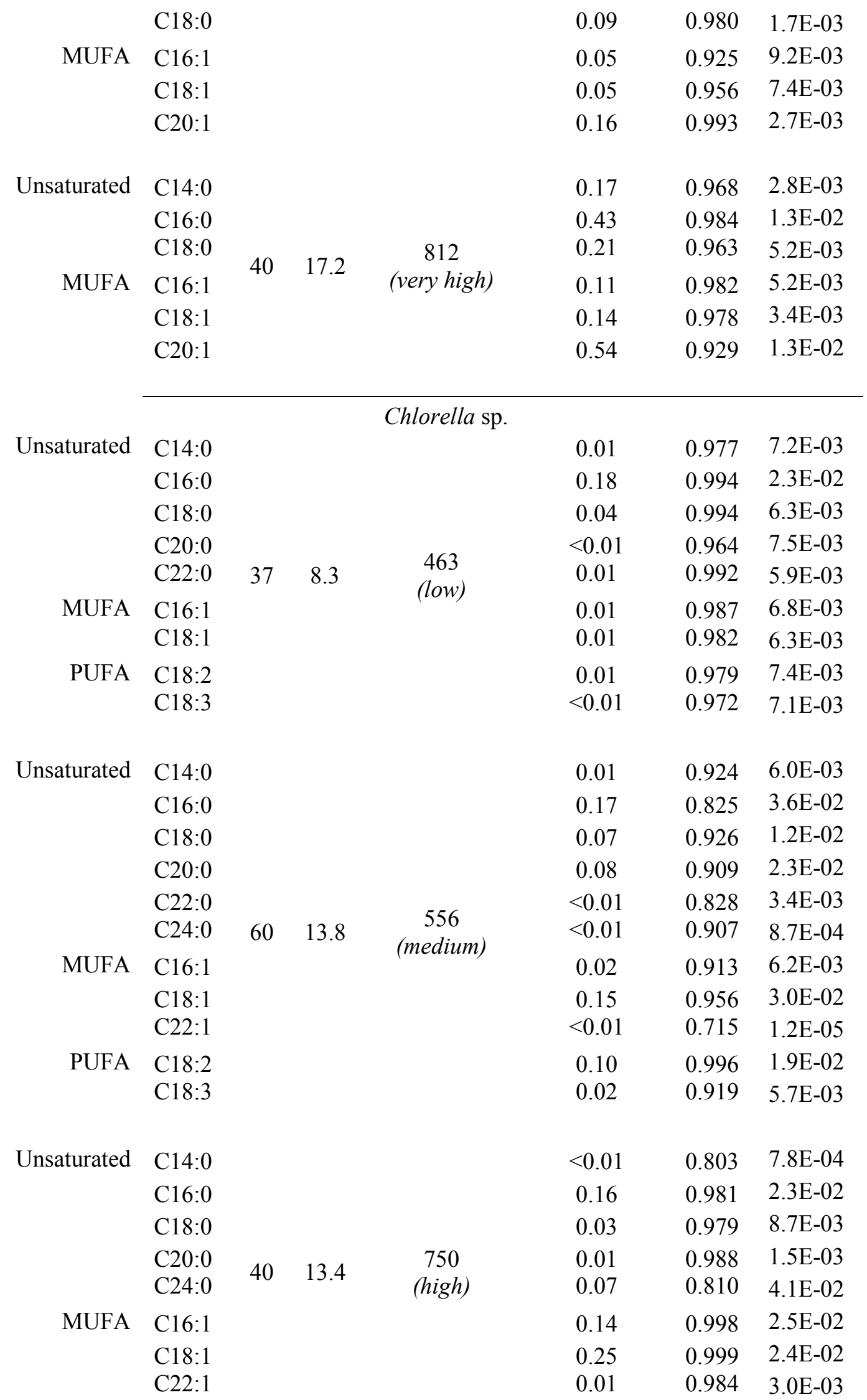


PUFA C18:2

$0.15 \quad 0.999 \quad 2.4 \mathrm{E}-02$

$\mathrm{C} 18: 3$

$0.17 \quad 0.993 \quad 3.2 \mathrm{E}-02$

Table S.3 Solubility separation factors for 6-component analytical standard and Chlorella sp. extracts at very low, low, medium, high and very high scCO2 densities.

Solubility Separation

\begin{tabular}{rccc} 
Factor & \multicolumn{3}{c}{ Multi-Component } \\
\cline { 2 - 4 } CO2 Density & $\begin{array}{r}\text { medium } \\
550 \mathrm{mg} / \mathrm{ml}\end{array}$ & $\begin{array}{c}\text { high } \\
750 \mathrm{mg} / \mathrm{ml}\end{array}$ & $\begin{array}{c}\text { very high } \\
812 \mathrm{mg} / \mathrm{ml}\end{array}$ \\
\cline { 2 - 4 } $\mathrm{C} 14: 0 / \mathrm{C} 16: 0$ & 1.54 & 1.53 & 0.40 \\
$\mathrm{C} 14: 0 / \mathrm{C} 16: 1$ & 0.52 & 2.15 & 1.58 \\
$\mathrm{C} 14: 0 / \mathrm{C} 18: 0$ & 2.10 & 1.29 & 0.80 \\
$\mathrm{C} 14: 0 / \mathrm{C} 18: 1$ & 1.06 & 2.18 & 1.18 \\
$\mathrm{C} 14: 0 / \mathrm{C} 20: 1$ & 0.49 & 0.71 & 0.31 \\
$\mathrm{C} 16: 0 / \mathrm{C} 16: 1$ & 0.34 & 1.40 & 3.99 \\
$\mathrm{C} 16: 0 / \mathrm{C} 18: 0$ & 1.37 & 0.84 & 2.02 \\
$\mathrm{C} 16: 0 / \mathrm{C} 18: 1$ & 0.69 & 1.42 & 2.98 \\
$\mathrm{C} 16: 0 / \mathrm{C} 20: 1$ & 0.32 & 0.46 & 0.79 \\
$\mathrm{C} 16: 1 / \mathrm{C} 18: 0$ & 4.06 & 0.60 & 0.51 \\
$\mathrm{C} 16: 1 / \mathrm{C} 18: 1$ & 2.04 & 1.02 & 0.67 \\
$\mathrm{C} 16: 1 / \mathrm{C} 20: 1$ & 0.94 & 0.33 & 0.20 \\
$\mathrm{C} 18: 0 / \mathrm{C} 18: 1$ & 0.50 & 1.69 & 1.48 \\
$\mathrm{C} 18: 0 / \mathrm{C} 20: 1$ & 0.23 & 0.55 & 0.39 \\
$\mathrm{C} 18: 1 / \mathrm{C} 20: 1$ & 0.46 & 0.33 & 0.26
\end{tabular}

Solubility Separation

Factor Chlorella sp.

\begin{tabular}{|c|c|c|c|}
\hline CO2 Density & $\begin{array}{c}\text { low } \\
463 \mathrm{mg} / \mathrm{ml} \\
\end{array}$ & $\begin{array}{c}\text { medium } \\
556 \mathrm{mg} / \mathrm{ml} \\
\end{array}$ & $\begin{array}{c}\text { high } \\
750 \mathrm{mg} / \mathrm{ml}\end{array}$ \\
\hline C14:0/C16:0 & 0.07 & 0.03 & 0.01 \\
\hline C14:0/C16:1 & 0.62 & 1.14 & 0.01 \\
\hline C14:0/C18:0 & 0.19 & 0.17 & 0.05 \\
\hline C14:0/C18:1 & 0.08 & 0.42 & 0.01 \\
\hline C14:0/C18:2 & 0.12 & 0.61 & 0.01 \\
\hline $\mathrm{C} 14: 0 / \mathrm{C} 18: 3$ & 0.69 & 2.16 & 0.01 \\
\hline C14:0/C20:0 & 0.16 & 2.36 & 0.36 \\
\hline $\mathrm{C} 14: 0 / \mathrm{C} 22: 0$ & 3.91 & 0.87 & 0.19 \\
\hline C14:0/C22:1 & - & 4.51 & 0.21 \\
\hline $\mathrm{C} 14: 0 / \mathrm{C} 24: 0$ & - & 3.53 & 0.03 \\
\hline $\mathrm{C} 16: 0 / \mathrm{C} 16: 1$ & 8.30 & 34.32 & 1.12 \\
\hline
\end{tabular}




\begin{tabular}{|c|c|c|c|}
\hline C16:0/C18:0 & 2.52 & 4.97 & 4.65 \\
\hline C16:0/C18:1 & 1.08 & 12.77 & 0.65 \\
\hline $\mathrm{C} 16: 0 / \mathrm{C} 18: 2$ & 1.59 & 18.45 & 1.05 \\
\hline C16:0/C18:3 & 9.18 & 65.08 & 0.96 \\
\hline C16:0/C20:0 & 2.14 & 71.08 & 30.54 \\
\hline $\mathrm{C} 16: 0 / \mathrm{C} 22: 0$ & 52.40 & 26.10 & 16.29 \\
\hline C16:0/C22:1 & - & 135.72 & 17.53 \\
\hline $\mathrm{C} 16: 0 / \mathrm{C} 24: 0$ & - & 106.08 & 2.32 \\
\hline $\mathrm{C} 16: 1 / \mathrm{C} 18: 0$ & 0.30 & 0.14 & 4.16 \\
\hline C16:1/C18:1 & 0.13 & 0.37 & 0.58 \\
\hline $\mathrm{C} 16: 1 / \mathrm{C} 18: 2$ & 0.19 & 0.54 & 0.94 \\
\hline C16:1/C18:3 & 1.11 & 1.90 & 0.86 \\
\hline $\mathrm{C} 16: 1 / \mathrm{C} 20: 0$ & 0.26 & 2.07 & 27.30 \\
\hline $\mathrm{C} 16: 1 / \mathrm{C} 22: 0$ & 6.32 & 0.76 & 14.56 \\
\hline C16:1/C22:1 & - & 3.95 & 15.67 \\
\hline $\mathrm{C} 16: 1 / \mathrm{C} 24: 0$ & - & 3.09 & 2.07 \\
\hline C18:0/C18:1 & 0.43 & 2.57 & 0.14 \\
\hline C18:0/C18:2 & 0.63 & 3.71 & 0.23 \\
\hline $\mathrm{C} 18: 0 / \mathrm{C} 18: 3$ & 3.65 & 13.09 & 0.21 \\
\hline C18:0/C20:0 & 0.85 & 14.30 & 6.57 \\
\hline C18:0/C22:0 & 20.83 & 5.25 & 3.50 \\
\hline C18:0/C22:1 & - & 27.30 & 3.77 \\
\hline $\mathrm{C} 18: 0 / \mathrm{C} 24: 0$ & - & 21.34 & 0.50 \\
\hline C18:1/C18:2 & 1.44 & 1.46 & 1.61 \\
\hline $\mathrm{C} 18: 1 / \mathrm{C} 18: 3$ & 5.19 & 5.10 & 1.48 \\
\hline C18:1/C20:0 & 5.60 & 5.57 & 46.77 \\
\hline $\mathrm{C} 18: 1 / \mathrm{C} 22: 0$ & 48.33 & 2.04 & 24.95 \\
\hline C18:1/C22:1 & - & 10.63 & 26.84 \\
\hline $\mathrm{C} 18: 1 / \mathrm{C} 24: 0$ & - & 8.31 & 3.55 \\
\hline $\mathrm{C} 18: 2 / \mathrm{C} 18: 3$ & 5.76 & 3.53 & 0.91 \\
\hline C18:2/C20:0 & 1.34 & 3.85 & 29.00 \\
\hline $\mathrm{C} 18: 2 / \mathrm{C} 22: 0$ & 1.43 & 1.41 & 15.47 \\
\hline $\mathrm{C} 18: 2 / \mathrm{C} 22: 1$ & - & 7.36 & 16.64 \\
\hline C18:2/C24:0 & - & 5.75 & 2.20 \\
\hline $\mathrm{C} 18: 3 / \mathrm{C} 20: 0$ & 0.23 & 0.40 & 31.71 \\
\hline $\mathrm{C} 18: 3 / \mathrm{C} 22: 0$ & 5.71 & 0.40 & 16.91 \\
\hline C18:3/C22:1 & - & 2.09 & 18.20 \\
\hline $\mathrm{C} 18: 3 / \mathrm{C} 24: 0$ & - & 1.63 & 2.41 \\
\hline $\mathrm{C} 20: 0 / \mathrm{C} 22: 0$ & 24.46 & 0.37 & 0.53 \\
\hline $\mathrm{C} 20: 0 / \mathrm{C} 22: 1$ & - & 1.91 & 0.57 \\
\hline $\mathrm{C} 20: 0 / \mathrm{C} 24: 0$ & - & 1.49 & 0.08 \\
\hline $\mathrm{C} 22: 0 / \mathrm{C} 22: 1$ & - & 5.20 & 1.08 \\
\hline $\mathrm{C} 22: 0 / \mathrm{C} 24: 0$ & - & 4.06 & 0.14 \\
\hline
\end{tabular}


C22:1/C24:0

0.78

0.13 